diameter of the meteoroid is less than one mean free path in the air for a molecule travelling at very high velocity. This result is valid in spite of the fact that on the average such a re-emitted molecule suffers a collision with a free air molecule after travelling a distance from the surface which is very short compared to the mean free path in the air. The essential cause is that these collisions frequently eject the reemitted molecules laterally at high speeds beyond the edges of the meteoroid. Both Levin and Öpik have claimed that a similar process operates for vapors emitted from the meteoroid. In the case in which large numbers of meteoric molecules are emitted from the surface it can be shown that these can accumulate to the point where they stop the air stream ahead of the meteoroid and form a shield which transfers the kinetic energy thus acquired at its leading surface via a temperature gradient established in that shield both to the laterally outflowing meteoric vapor and to the surface of the meteoroid. The claim of Levin and of Öpik is justified for extremely slow meteors moving at speeds near $I 2 \mathrm{~km} / \mathrm{sec}$. At very high velocities near $72 \mathrm{~km} / \mathrm{sec}$ the vaporizing meteoroid's diameter must be less than one twentieth of a mean free path of a very high velocity meteoric molecule in air in order that shielding by its own vapors shall be negligible. The dependence of the heat transfer coefficient on diameter of the vaporizing meteoroid, in the range in which shielding by its own vapors is significant while shielding by an air cap is negligible, runs approximately as the inverse 0.4 power.

\section{Harvard College Observatory} Cambridge, Mass.

Cook, A. F. II, Hawkins, G. S. and Steinon, F. M. The width of meteor trails II.

A detailed comparison is made between the images of meteor trails and star trails. The photographs were obtained by A. F. Cook during the Geminid shower of 1957 using the 48-inch Schmidt telescope on Mt. Palomar. Adjustments were made in the focal setting of the telescope to allow for the fact that the meteors were not at infinity. Microdensitometer tracings showed that the intensity distribution in the images was gaussian and the widths were measured at the point where the intensity had decreased by a factor of $\mathrm{I} / e$. A significant dif- ference in width was found between in-focus meteor images and the in-focus star images. The average width of the Geminid meteors was found to be 3 meters and the average magnitude was +4 . One meteor, magnitude $\mathrm{o}$, showed a width considerably in excess of this value, splitting into three fragments which had separated by as much as 20 meters at the end of the trail. A trail width of several meters is inconsistent with the assumption that meteors are small solid bodies. It is consistent with the dustball hypothesis in which severe fragmentation takes place as the meteor body is disrupted in the upper atmosphere.

Harvard College Observatory Cambridge, Mass.

\section{Cuffey, James. $N G C 6838$.}

NGC 6838 is one of the richest of the clusters listed in the catalogues of galactic clusters. Because the brightest stars were red and the fainter ones bluer, it was suspected by many to be a globular cluster. Since it is strategically located near the direction toward which the stars in the solar neighborhood are moving under galactic rotation, it is especially desirable that its true nature be known.

A study of the color-magnitude diagram, based on plates taken with the roo-inch $\mathrm{Mt}$. Wilson reflector and photoelectric standards observed with the 82-inch McDonald telescope, shows that although the brightest stars are red and the fainter stars become bluer with decreasing brightness, the cluster is not a typical globular. The red giant branch is characterized by wide scatter and is poorly defined. The horizontal white branch is missing, and no very blue stars are present. No cluster-type variables are present. Thus, the cluster lacks most of the attributes now regarded as appropriate to the color-magnitude relation of a typical globular cluster.

Goethe Link Observatory Indiana University Bloomington, Ind.

Demarque, Pierre. The structure of subdwarf stars.

It is generally accepted that the subdwarfs are characterized by a low metal abundance. The present investigation indicates that the helium content also plays a role in differentiating among subdwarfs. 